\title{
Systematic review of telemedicine in spine surgery
}

\author{
*John Paul G. Kolcun, MD, Won Hyung A. Ryu, MD, MS, MTM, and Vincent C. Traynelis, MD \\ Department of Neurological Surgery, Rush University Medical Center, Chicago, Illinois
}

OBJECTIVE The use of telemedicine (TM) has long been available, but recent restrictions to hospitals due to the coronavirus disease 2019 (COVID-19) pandemic have accelerated the global implementation of TM. However, evidence on the effectiveness of this technology for the care of spine surgery patients is limited. In this systematic review the authors aimed to examine the current utilization of TM for spine surgery.

METHODS Using PubMed, Scopus, and the Cochrane Library, the authors performed a systematic review of the literature focused on the themes of telemedicine and spine surgery. Included in the search were randomized controlled trials, cohort studies, and case-controlled studies. Two independent reviewers conducted the study appraisal, data abstraction, and quality assessments of the studies.

RESULTS Out of 1463 references from the initial search results, 12 studies met the inclusion criteria. The majority of TM interventions focused on improving perioperative patient communication and patient education by using mobile phone apps, online surveys, or online materials for consent. The studies reported the feasibility of the use of TM for perioperative care and positive user experiences from the patients.

CONCLUSIONS The current increase in TM adoption due to the COVID-19 crisis presents an opportunity to further develop and validate this technology. Early evidence in the literature supports the use of TM as an adjunct to traditional in-person clinical encounters for certain perioperative tasks such as supplemental patient education and postoperative surveys.

https://thejns.org/doi/abs/10.3171/2020.6.SPINE20863

KEYWORDS telemedicine; systematic review; spine surgery

$\mathrm{W}$ ITH increased access to modern communications technology, telemedicine (TM) has seen an appreciable rise in clinical practice over recent decades in a variety of medical specialties. In turn, many studies have investigated those factors associated with cost-effective implementation and patient satisfac-

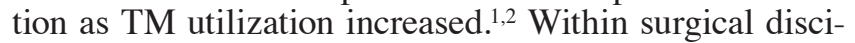
plines, however, remote encounters are often not feasible or practical, as clinic visits often involve physical interactions (i.e., neurological examinations, wound checks, or inclinic procedures). This limitation is particularly relevant in spine surgery, where management decisions often hinge on subtle physical examination findings and an in-depth and more nuanced discussion with the patient than usually occurs in a TM setting. In addition, administrative and legal concerns such as inconsistent insurance coverage, liability in managing patients at a distance, and crossstate licensure remain major barriers for TM. For example, reimbursement policies have lagged behind technological advancement: as recently as 2016, only 29 states within the United States had private insurance coverage for TM visits. ${ }^{3}$ Together, these clinical, legal, and financial limitations have prevented the wide adoption of TM in the management of patients with spinal pathology.

The recent coronavirus disease 2019 (COVID-19) emergency has dramatically altered practices across surgical specialties, including spine surgery. Government and hospital policies in response to this pandemic have not only limited surgery case volumes but also accessibility to outpatient clinics. While these guidelines with increased

ABBREVIATIONS COVID-19 = coronavirus disease 2019; ODI = Oswestry Disability Index; PCS = physical component summary; PODCI = Pediatric Outcomes Data Collection Instrument; PRO = patient-reported outcome; RCT = randomized controlled trial; SRS = Scoliosis Research Society; TM = telemedicine; VAS = visual analog scale. SUBMITTED May 12, 2020. ACCEPTED June 17, 2020.

INCLUDE WHEN CITING Published online October 30, 2020; DOI: 10.3171/2020.6.SPINE20863.

* J.P.G.K. and W.H.A.R. contributed equally to this study. 
restrictions to hospitals are necessary, they have also created significant challenges in ensuring continuity of patient care for new surgical consultations and postoperative follow-ups. One of the most commonly implemented solutions to addressing access to care has been the use of TM. However, the most appropriate means of incorporating TM into clinical practice remain under debate.,.,5

To better inform this conversation, we performed a systematic literature review of TM apps in spine surgery prior to the COVID-19 pandemic, in order to contextualize the rapid adoption of TM practices necessitated by this crisis.

\section{Methods}

\section{Search Strategy and Study Selection}

Our systematic review strictly adhered to the PRISMA guidelines. ${ }^{6}$ Our study protocol was registered in advance with the International Prospective Register of Systematic Reviews.

We performed a systematic literature review drawing from the PubMed, Cochrane Library, and Scopus databases without time/date restriction. Search strings included a variety of MeSH keywords relating to TM in combination with MeSH keywords related to spine surgery (see Appendix). Subsequently, an additional search was run using Google Scholar and drawing from references in review articles identified by our initial search. Search results were screened and reviewed sequentially by title, abstract, and, finally, full content by two independent reviewers (J.P.G.K., W.H.A.R.).

\section{Inclusion and Exclusion Criteria}

TM was defined as any form of intervention or communication via online, digital, or phone-based platforms between spine surgery patients and their surgeons or clinical staff. For the purposes of this investigation, we targeted studies relating to the application of TM interventions in patients with spine or spinal cord pathology involving surgical care. We included randomized controlled trials (RCTs), cohort studies, and case-control studies, and excluded case reports, editorials, technical reports, conference abstracts, books, and non-English-language studies. We also excluded studies involving the use of TM for patients with spinal cord injury, given that several systematic reviews of this topic have recently been published.

\section{Data Extraction and Analysis}

Study data were extracted by two independent reviewers (J.P.G.K., W.H.A.R.) using a standard collection form developed prior to our literature search. Data fields included author, publication year, journal of publication, country, study design, sample size, intervention (e.g., phone-based communication, online-based communication, mobile phone app, etc.), primary outcomes (e.g., patient compliance/adherence, perception of TM intervention, clinical metrics), comparator group (if applicable), results, funding sources, and study limitations.

Given the exploratory nature of our study questions, meta-analysis was not performed. Rather, studies relating to TM applications in spinal surgery patients were organized based on type of patient care (e.g., preopera- tive management, postoperative follow-up). The included studies were also reviewed according to the nature of the intervention (e.g., phone surveys, mobile apps), primary outcome, and study design. In this way, the authors were able to summarize relevant literature in a structured, descriptive fashion.

Two independent reviewers (J.P.G.K., W.H.A.R.) evaluated the quality of appropriate study types (e.g., RCTs, prospective cohort studies) which met inclusion criteria using the National Institutes of Health (NIH) Study Quality Assessment Tools. ${ }^{8}$ These tools are standardized questionnaires ranging from 12 to 14 items (based on study type) that rate study quality as good, fair, or poor. Score discrepancies between reviewers were resolved through discussion and joint review.

\section{Results \\ Search Results}

Our initial searches yielded 1463 results (403 in PubMed, 440 in Cochrane, and 607 in the Scopus databases, and 13 from the Google Scholar search engine) prior to any screening. Initial screening of our search results identified 65 unique studies meeting our inclusion criteria. Further review of abstracts left 21 studies remaining which met inclusion criteria. Full analysis excluded a further 9 studies, leaving 12 total studies which met criteria for final inclusion. The literature review and study selection process are summarized in Fig. 1.9 The selected studies included 4 RCTs, 4 prospective cohort studies, 2 randomized crossover studies, 1 prospective observational study, and 1 cost-efficacy cohort study. Publication dates ranged from 2016 to 2019. Studies were conducted in North America (7), Europe (3), and Asia (2). Three studies included general neurosurgery cohorts, while the remaining studies were strictly spine patients (Table 1).

\section{Overview of Interventions and Outcomes}

In general, a majority of TM interventions (7 of 12) facilitated communication between patients and surgeons' office staff, in the form of mobile phone apps for patients to self-report symptoms or functional status, online surveys, phone surveys, or online materials for education and consent. ${ }^{10-16}$ Two studies described internet-based systems with postoperative rehabilitation/exercise instructions in lieu of in-person physical therapy visits. ${ }^{17,18}$ One study described virtual TM postoperative visits in the place of inperson clinic visits. ${ }^{19}$ One study evaluated patient participation in an online support group for patients undergoing lumbar fusion..$^{20}$ Finally, one study assessed a wearable accelerometer device to measure physical activity in relation to patient-reported outcomes (PROs). ${ }^{21}$

Studies with a comparator arm included analyses for the efficacy of the TM intervention relative to the control group, including patient compliance with the TM intervention, preference, and impact on target outcomes (Tables 2 and 3). As the majority of studies described TM systems for communication and patient interview, target outcomes generally dealt with information exchange between surgeons and patients. These outcomes included both patient compliance with the TM applications and differences in 


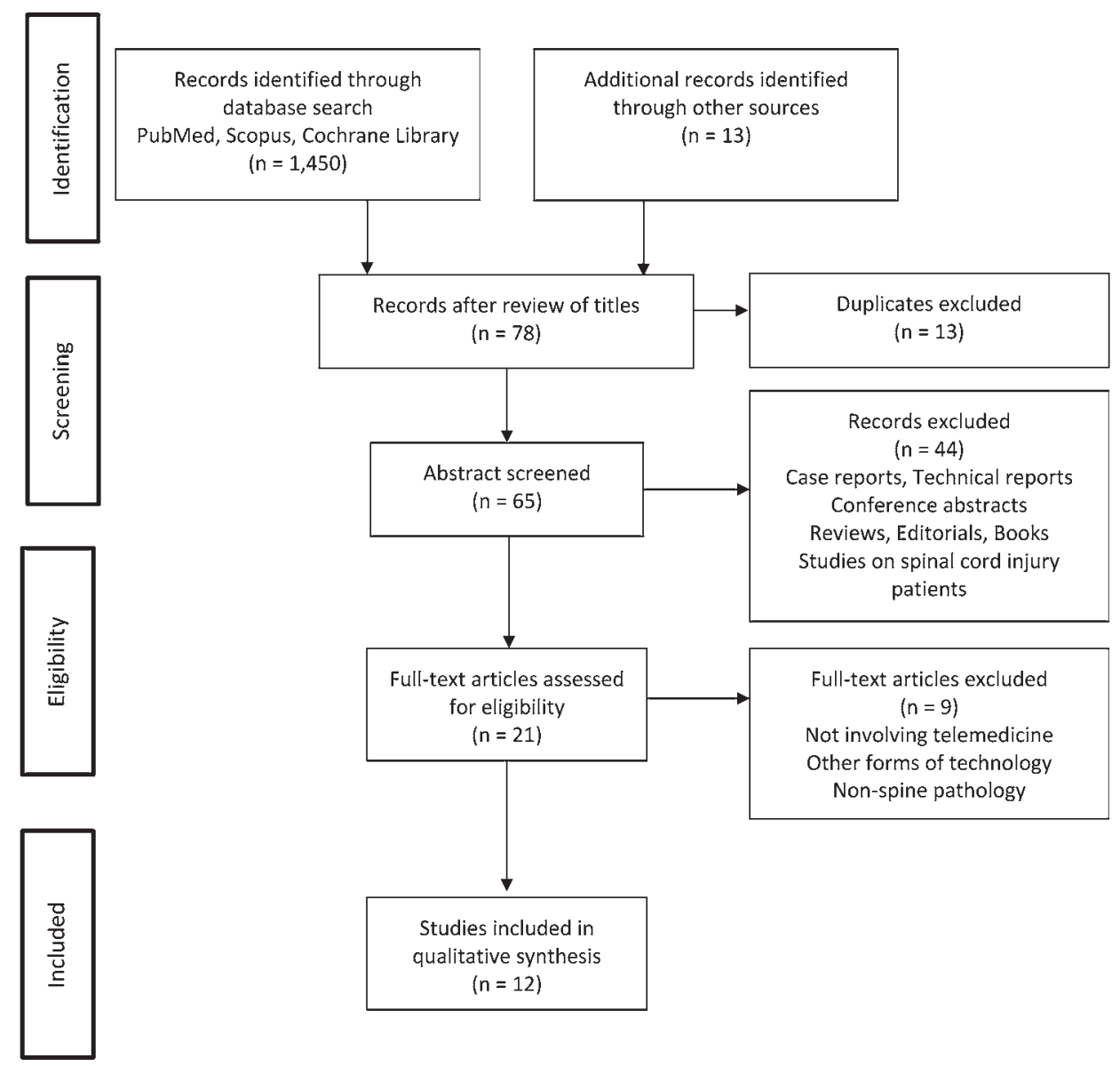

FIG. 1. Flowchart of the systematic search strategy and study patient selection.

PROs or other clinical metrics between conventional and TM groups.

\section{TM for PRO Surveys}

Bokshan et al. ${ }^{11}$ assessed the reliability of Scoliosis Research Society-22r (SRS-22r) and Oswestry Disability Index (ODI) results when administered to adult deformity patients over the phone as compared to patients surveyed in person in a prospective randomized crossover study. They found no significant difference between phone and in-person PROs, with a high degree of score correlation within groups. Telephone was the preferred form of survey for $57 \%$ of study patients. Nitikman et al. ${ }^{14}$ similarly tested online administration of the SRS-30 and Pediatric Outcomes Data Collection Instrument (PODCI) in 96 adolescents with idiopathic scoliosis randomized to internetbased versus paper-based survey arms. There were no significant differences in survey results between groups, with $84 \%$ of patients preferring online administration.

\section{TM for Preoperative Care}

Stewart et al..$^{15}$ investigated the impact on surgery cancellations of patient use of a mobile app with preoperative instructions and notifications. This study included 174 patients undergoing elective spine surgery. Primary out- comes were overall adherence to preoperative instructions and case cancellation rate. There were no surgical cancellations in the TM group (85 patients), while $5.6 \%$ of patients in the comparison group had cancelled surgeries due to patient noncompliance with preoperative instructions $\left(5 / 89\right.$ patients). Bethune et al. ${ }^{10}$ evaluated a multimedia online educational tool for preoperative education regarding disease and surgical consent in 38 neurosurgical patients (including patients with degenerative spine and intracranial pathologies). Patients were randomized between the online educational tool versus a standard informed consent process. Efficacy was measured by a 14-item questionnaire to assess patients' understanding of disease, surgical approach, prognosis, and postoperative course. Patients in the TM educational group scored significantly higher on the questionnaire.

\section{TM for Postoperative Care}

Debono et al. ${ }^{12}$ described 60 patients undergoing lumbar discectomy who were given a mobile app with daily postoperative questionnaires regarding pain, temperature, neurological symptoms, and wound status for 15 days, in addition to a telephone call on postoperative day 1. At 3 months postoperatively, patients were surveyed regarding their overall experience and opinion of the application 
TABLE 1. Characteristics of the studies included in the systematic review

\begin{tabular}{|c|c|c|c|}
\hline Authors \& Year & Journal & $\begin{array}{l}\text { Location } \\
\text { (country) }\end{array}$ & Funding Source \\
\hline Bethune et al., $2018^{10}$ & Canadian Journal of Surgery & Canada & $\begin{array}{l}\text { SHS-13-009 from Academic Health Sciences Centre Alternative Funding Plan } \\
\text { Innovation Fund }\end{array}$ \\
\hline Bokshan et al., $2016^{11}$ & Spine Journal & USA & CTSA program of NIH NCATS, awards TL1 TR000449 \& UL1 TR000448 \\
\hline Debono et al., $2016^{12}$ & European Spine Journal & France & NR \\
\hline Dicianno et al., $2016^{17}$ & $\begin{array}{l}\text { American Journal of } \\
\text { Physical Medicine \& } \\
\text { Rehabilitation }\end{array}$ & USA & $\begin{array}{l}\text { NIDILRR grant 90DP5004-01-00 (RERC from Cloud to Smartphone: Empowering \& } \\
\text { Accessible ICT), grant 0DP0064-01-00 (DRRP promoting independence \& self- } \\
\text { management using mHealth), \& grant 90RE5004-01-00 (RERC on telerehabilita- } \\
\text { tion), Verizon Foundation, \& NIH grants 1R21HD071810-01A1 \& 5T35AT005933-02 }\end{array}$ \\
\hline Felbaum et al., $2018^{13}$ & Operative Neurosurgery & USA & NR \\
\hline Hou et al., $2019^{18}$ & $\begin{array}{l}\text { Journal of Medical Internet } \\
\text { Research mHealth \& } \\
\text { uHealth }\end{array}$ & China & $\begin{array}{l}\text { Guangdong Medical Research Fund Project (C2015048) \& Guangdong Natural } \\
\text { Science Foundation (2015A030310321) }\end{array}$ \\
\hline Nitikman et al., $2017^{14}$ & $\begin{array}{l}\text { Journal of Pediatrics \& } \\
\text { Orthopedics }\end{array}$ & Canada & NR \\
\hline Scheer et al., $2017^{21}$ & Clinical Spine Surgery & USA & $\begin{array}{l}\text { Scholarship support for } 1 \text { author (Alpha Omega Alpha Carolyn L. Kuckein Student } \\
\text { Research Fellowship) }\end{array}$ \\
\hline Stewart et al., $2019^{15}$ & Cureus & USA & NR \\
\hline Strøm et al., $2019^{16}$ & Spine Journal & Denmark & $\begin{array}{l}\text { Helsefonden (independent charity), Health Research Fund of Jutland Hospital, } \\
\text { Toyota Foundation }\end{array}$ \\
\hline Strøm et al., $2019^{20}$ & $\begin{array}{l}\text { Journal of Medical Internet } \\
\text { Research }\end{array}$ & Denmark & NR \\
\hline Thakar et al., $2018^{19}$ & Neurosurgical Focus & India & NR \\
\hline
\end{tabular}

CTSA = Clinical and Translational Science Award; DRRP = Disability and Rehabilitation Research Projects; ICT = information and communications technology; NCATS = National Center for Advancing Translational Sciences; NIDILRR = National Institute on Disability, Independent Living, and Rehabilitation Research; NR = not reported; RERC $=$ rehabilitation engineering research center.

(rating scale 1-4). A majority of patients did use the app $(55 / 60)$, with a high average satisfaction rate (3.5/4). There were 29 "alarms" triggered by the app that required contact by the neurosurgeon's office. The majority of these alarms were for pain management $(72.4 \%)$. Other reasons included mild fever, voiding delay, and functional/mobility questions. Hou et al. ${ }^{18}$ randomized 168 patients undergoing elective spine surgery for degenerative disease to postoperative rehabilitation instructions/communication via mobile app versus standard care with physical activity recommendations at discharge. Primary outcomes included ODI and pain visual analog scale (VAS) score compared between groups. At 2 years postoperatively, the TM cohort had significantly greater improvements in both ODI and pain severity.

Thakar et al..$^{19}$ performed a cost-effectiveness analysis of virtual TM postoperative visits in a general neurosurgery population with a total of 1200 patients, including spine patients, between two sites in India (one in-person clinic and one major center housing the TM practice). The authors concluded that TM visits were far superior from an economic perspective, at approximately one-third the average cost of in-person clinic visits. This model was sustainable even if $50 \%$ of the TM visits were failures requiring subsequent in-person evaluation.

\section{TM for General Clinical Care}

In a study by Dicianno et al., ${ }^{17}$ patients with myelome- ningocele and hydrocephalus from an adult spina bifida clinic were randomly assigned to a group using a mobile app with modules for medication management, self-care schedules, and a clinical portal for 2-way communication or a group using standard care from the same clinic. Outcomes included app usage, assessments of physical and self-management independence, depression, and quality of life. Across the study groups, there were no significant differences in these outcomes. Similarly, there were no differences in medical events (e.g., emergency room visits, urinary tract infections). A subset of patients deemed "high-volume users" of the app, however, did have higher scores for self-management and independence. Felbaum et al. ${ }^{13}$ reported the use of a mobile app for preoperative instructions and postoperative evaluation in 56 patients undergoing elective neurosurgical procedures (55/56 spine procedures). Primary outcomes included successful installation and registration with the app, compliance with reading instructions through the app, and successful communication of pain scores and wound images postoperatively. A vast majority of patients successfully obtained the app and used it postoperatively (54/56). There were no cancellations attributed to noncompliance with preoperative instructions. There was one postoperative complication (wound infection) detected through the app. While this was a small pilot study without a comparator group, the authors believe it shows promise for the perioperative use of this TM intervention. 
TABLE 2. Design of the studies included in the systematic review

\begin{tabular}{|c|c|c|c|c|c|c|}
\hline $\begin{array}{c}\text { Authors } \\
\text { \& Year }\end{array}$ & $\begin{array}{l}\text { Study } \\
\text { Design }\end{array}$ & $\begin{array}{c}\text { Study } \\
\text { Objective }\end{array}$ & $\begin{array}{c}\text { Pt } \\
\text { Population }\end{array}$ & Intervention & Comparator & $\begin{array}{l}\text { Primary } \\
\text { Outcome }\end{array}$ \\
\hline $\begin{array}{l}\text { Bethune } \\
\text { et al., } \\
2018^{10}\end{array}$ & RCT & $\begin{array}{l}\text { Evaluate multimedia educa- } \\
\text { tional tool w/ interactive } \\
\text { booklets to enhance pt } \\
\text { knowledge before decision } \\
\text { to undergo op }\end{array}$ & $\begin{array}{l}\text { Degenerative } \\
\text { spine disease } \\
\text { (cervical \& } \\
\text { lumbar), brain } \\
\text { tumor, trigemi- } \\
\text { nal neuralgia }\end{array}$ & $\begin{array}{l}\text { e-book w/ info on disease } \\
\& \text { standard consent } \\
\text { process }\end{array}$ & $\begin{array}{l}\text { Standard } \\
\text { consent } \\
\text { process }\end{array}$ & $\begin{array}{l}\text { 14-item questionnaire re } \\
\text { pt understanding of dis- } \\
\text { ease, op approach, risk, } \\
\text { recovery expectation, } \\
\text { postop pain, alternative } \\
\text { options }\end{array}$ \\
\hline $\begin{array}{l}\text { Bokshan } \\
\text { et al., } \\
2016^{11}\end{array}$ & $\begin{array}{l}\text { Randomized } \\
\text { crossover } \\
\text { validation } \\
\text { study }\end{array}$ & $\begin{array}{l}\text { Examine reliability of } \\
\text { SRS-22r \& ODI question- } \\
\text { naires administered by } \\
\text { phone in ASD pts }\end{array}$ & ASD & $\begin{array}{l}\text { Phone survey of SRS-22r } \\
\quad \& \text { ODI }\end{array}$ & $\begin{array}{l}\text { In-person pt } \\
\text { survey }\end{array}$ & ODI \& SRS-22r surveys \\
\hline $\begin{array}{l}\text { Debono } \\
\text { et al., } \\
2016^{12}\end{array}$ & $\begin{array}{l}\text { Prospective } \\
\text { cohort } \\
\text { study }\end{array}$ & $\begin{array}{l}\text { Assess mobile app feasibility } \\
\text { for postop monitoring after } \\
\text { outpatient lumbar disc- } \\
\text { ectomy }\end{array}$ & $\begin{array}{l}\text { Lumbar discec- } \\
\text { tomy }\end{array}$ & $\begin{array}{l}\text { Mobile app w/ daily } \\
\text { questionnaire (pain, } \\
\text { temperature, neurologic } \\
\text { symptoms, wound status) } \\
\text { until } 15 \text { days postop for } \\
\text { monitoring along w/ stan- } \\
\text { dard postop day } 1 \text { phone } \\
\text { call \& 6-wk clinic visit }\end{array}$ & None & $\begin{array}{l}\text { Pt survey at } 3 \text { mos postop } \\
\text { focusing on pt recovery } \\
\text { experience \& user } \\
\text { experience of mobile } \\
\text { app (VAS score 1-4) }\end{array}$ \\
\hline $\begin{array}{l}\text { Dicianno } \\
\text { et al., } \\
2016^{17}\end{array}$ & RCT & $\begin{array}{l}\text { In pts w/ myelomeningocele: } \\
\text { determine 1) if mobile app } \\
\text { use improves pt medical \& } \\
\text { psychosocial outcomes; 2) } \\
\text { if pts consistently use } \\
\text { self-management system }\end{array}$ & $\begin{array}{l}\text { Adults } w / \text { myelo- } \\
\text { meningocele } \\
\text { w/ hydro- } \\
\text { cephalus }\end{array}$ & $\begin{array}{l}\text { Mobile app (mobile health } \\
\text { \& rehab system: iMHere) } \\
\text { to support self-man- } \\
\text { agement of adults w/ } \\
\text { disability; modules for } \\
\text { medication, self-care } \\
\text { schedule, web-based } \\
\text { clinician portal, 2-way } \\
\text { communication system }\end{array}$ & $\begin{array}{l}\text { Standard care } \\
\text { from adult } \\
\text { spina bifida } \\
\text { clinic }\end{array}$ & $\begin{array}{l}\text { Mobile app to assess } \\
\text { physical independence, } \\
\text { self-management skill, } \\
\text { depression, \& quality } \\
\text { of life }\end{array}$ \\
\hline $\begin{array}{l}\text { Felbaum } \\
\text { et al., } \\
2018^{13}\end{array}$ & $\begin{array}{l}\text { Prospective } \\
\text { cohort } \\
\text { study }\end{array}$ & $\begin{array}{l}\text { Demonstrate app-based } \\
\text { instructions w/ built-in } \\
\text { reminders to improve pt } \\
\text { understanding \& compli- } \\
\text { ance, contribute to reduc- } \\
\text { ing no. of op cancellations, } \\
\text { postop complications, \& } \\
\text { readmissions }\end{array}$ & $\begin{array}{l}\text { Pts undergo- } \\
\text { ing elective } \\
\text { neurosurgery } \\
\text { procedures } \\
\text { (55/56 spine } \\
\text { procedures) }\end{array}$ & $\begin{array}{l}\text { Mobile app (TrackMyRe- } \\
\text { covery) for pre- \& postop } \\
\text { follow-up }\end{array}$ & None & $\begin{array}{l}\text { 1) Successful registration } \\
\text { \& use of app; 2) } \\
\text { compliance w/ reading } \\
\text { instructions pre- \& } \\
\text { postop; 3) sending pain } \\
\text { scores \&/or wound } \\
\text { images }\end{array}$ \\
\hline $\begin{array}{l}\text { Hou } \\
\text { et al., } \\
2019^{18}\end{array}$ & RCT & $\begin{array}{l}\text { Examine efficacy of mobile } \\
\text { phone-based rehab } \\
\text { systems in pts who } \\
\text { underwent lumbar } \\
\text { spinal op }\end{array}$ & $\begin{array}{l}\text { Pts undergo- } \\
\text { ing elective } \\
\text { spine op for } \\
\text { degenerative } \\
\text { disc disease }\end{array}$ & $\begin{array}{l}\text { Mobile app w/ rehab } \\
\text { instructions \& as tool to } \\
\text { communicate w/ physi- } \\
\text { cian }\end{array}$ & $\begin{array}{l}\text { Standard care } \\
\text { w/ postop } \\
\text { instructions } \\
\text { for physical } \\
\text { activity }\end{array}$ & $\begin{array}{l}\text { 1) Function \& pain status } \\
\text { assessed by ODI; 2) } \\
\text { VAS }\end{array}$ \\
\hline $\begin{array}{l}\text { Nitikman } \\
\text { et al., } \\
2017^{14}\end{array}$ & $\begin{array}{l}\text { Randomized } \\
\text { crossover } \\
\text { validation } \\
\text { study }\end{array}$ & $\begin{array}{l}\text { Validate \& test reliability of } \\
\text { internet for administering } \\
\text { HRQOL questionnaires } \\
\text { (SRS-30 \& PODCI adoles- } \\
\text { cent self-report question- } \\
\text { naire) to adolescents w/ } \\
\text { idiopathic scoliosis }\end{array}$ & $\begin{array}{l}\text { Adolescents (age } \\
11-18 \text { yrs) } \\
\text { w/ idiopathic } \\
\text { scoliosis }\end{array}$ & $\begin{array}{l}\text { Internet-based SRS-30 \& } \\
\text { PODCI surveys }\end{array}$ & $\begin{array}{c}\text { Paper-based } \\
\text { pt survey }\end{array}$ & SRS-30 \& PODCI surveys \\
\hline $\begin{array}{l}\text { Scheer } \\
\text { et al., } \\
2017^{21}\end{array}$ & $\begin{array}{l}\text { Prospective } \\
\text { obser- } \\
\text { vational } \\
\text { study }\end{array}$ & $\begin{array}{l}\text { Evaluate accelerometer- } \\
\text { based device to measure } \\
\text { postop physical activity } \\
\text { correlates w/ postop PROs }\end{array}$ & Spine deformity & $\begin{array}{l}\text { 3D accelerometer device to } \\
\text { measure activity }\end{array}$ & NA & $\begin{array}{l}\text { Correlation of physical } \\
\text { activity measured w/ } \\
\text { monitor device (step } \\
\text { count, hourly step rate, } \\
\text { activity intensity) w/ } \\
\text { PROs }\end{array}$ \\
\hline
\end{tabular}


" CONTINUED FROM PAGE 165

TABLE 2. Design of the studies included in the systematic review

\begin{tabular}{|c|c|c|c|c|c|c|}
\hline $\begin{array}{l}\text { Authors } \\
\& \text { Year }\end{array}$ & $\begin{array}{l}\text { Study } \\
\text { Design }\end{array}$ & $\begin{array}{c}\text { Study } \\
\text { Objective }\end{array}$ & $\begin{array}{c}\text { Pt } \\
\text { Population }\end{array}$ & Intervention & Comparator & $\begin{array}{l}\text { Primary } \\
\text { Outcome }\end{array}$ \\
\hline $\begin{array}{l}\text { Stewart } \\
\text { et al., } \\
2019^{15}\end{array}$ & $\begin{array}{l}\text { Prospective } \\
\text { cohort } \\
\text { study }\end{array}$ & $\begin{array}{l}\text { Determine if mobile phone } \\
\text { app preop instructions \& } \\
\text { notifications 1) increase pt } \\
\text { compliance w/ instruc- } \\
\text { tions; 2) reduce case } \\
\text { cancellations }\end{array}$ & Elective spine op & $\begin{array}{l}\text { Mobile app (Amie, } \\
\text { FavorHealth Inc.) to } \\
\text { deliver preop } \\
\text { instructions, reminders, } \\
\text { \& notifications }\end{array}$ & Pts w/o app & $\begin{array}{l}\text { 1) Compliance w/ preop } \\
\text { instructions; } 2 \text { ) case } \\
\text { cancellation w/in } 48 \mathrm{hrs} \\
\text { of scheduled op due to } \\
\text { noncompliance }\end{array}$ \\
\hline $\begin{array}{l}\text { Strøm } \\
\text { et al., } \\
2019^{16}\end{array}$ & RCT & $\begin{array}{l}\text { Assess anxiety/depression } \\
\text { decrease in lumbar spinal } \\
\text { fusion pts w/ online sup- } \\
\text { port group participation }\end{array}$ & $\begin{array}{l}\text { Lumbar spine } \\
\text { fusion }\end{array}$ & $\begin{array}{l}\text { Online info platform \& sup- } \\
\text { port group (w-SPIINA) }\end{array}$ & $\begin{array}{l}\text { Standard } \\
\text { information } \\
\text { regimen }\end{array}$ & $\begin{array}{l}\text { Baseline to 3-mo postop } \\
\text { change in HADS }\end{array}$ \\
\hline $\begin{array}{l}\text { Strøm } \\
\text { et al., } \\
2019^{20}\end{array}$ & $\begin{array}{l}\text { Prospective } \\
\text { obser- } \\
\text { vational } \\
\text { study }\end{array}$ & $\begin{array}{l}\text { Determine socioeconomic/ } \\
\text { demographic characteris- } \\
\text { tics predicting participation } \\
\text { in online support group for } \\
\text { lumbar spine fusion pts }\end{array}$ & $\begin{array}{l}\text { Lumbar spine } \\
\text { fusion }\end{array}$ & $\begin{array}{l}\text { Online info platform \& sup- } \\
\text { port group }\end{array}$ & None & $\begin{array}{l}\text { Describe socioeconomic/ } \\
\text { demographic } \\
\text { characteristics of pts } \\
\text { using online support } \\
\text { group }\end{array}$ \\
\hline $\begin{array}{l}\text { Thakar } \\
\text { et al., } \\
2018^{19}\end{array}$ & $\begin{array}{l}\text { Retrospective } \\
\text { decision } \\
\text { analytical } \\
\text { model }\end{array}$ & $\begin{array}{l}\text { Determine cost-effectiveness } \\
\text { of remote virtual neuro- } \\
\text { surgery postop clinic visits } \\
\text { compared w/ conventional } \\
\text { in-person clinic visits }\end{array}$ & $\begin{array}{l}\text { General neuro- } \\
\text { surgery pts, } \\
\text { including spine }\end{array}$ & Virtual TM postop visits & $\begin{array}{l}\text { Standard } \\
\text { in-person } \\
\text { postop } \\
\text { visits }\end{array}$ & $\begin{array}{l}\text { 1) \% TM visits successful; } \\
\text { 2) pt-perceived utility } \\
\text { for overall experience; } \\
\text { 3) ICER }\end{array}$ \\
\hline
\end{tabular}

$\mathrm{ASD}=$ adult spinal deformity; HADS = Hospital Anxiety and Depression Scale; HRQOL = health-related quality of life; ICER = incremental cost-effectiveness ratio; NA = not applicable; $p t=$ patient; $w$-SPIINA = web-based Spine Platform featuring Interaction and Information by Animation.

\section{Other TM Interventions}

Strøm et al. ${ }^{16}$ investigated a cohort of spine patients who engaged in an online support group for patients undergoing lumbar fusion. In one study, the authors compared psychological outcomes (anxiety/depression), as measured by a change in an anxiety/depression scale at 3 months postoperatively, between 114 lumbar fusion patients randomized to use an online informational platform versus standard information/care. ${ }^{16}$ While there was not a significant difference between groups, there was a high compliance rate among online users. Given the small sample size, the authors suggest there may still be some benefit to this online community not revealed by this study. In a subsequent subanalysis of patients who used the online tool, Strøm et al. tried to identify sociodemographic or clinical characteristics predicting increased activity in the online support group. ${ }^{20}$ The authors found that female patients were significantly more likely to engage with the online tool. Further, patients' posts fell into 7 distinct thematic categories (e.g., activity/rehabilitation, postoperative pain, employment).

Scheer et al. ${ }^{21}$ assessed a wearable physical activity monitor in 22 deformity patients and attempted to correlate postoperative objective physical activity measurements with simultaneous PROs at 6 months, including the ODI and SF-36 physical component summary (PCS). There was a significant correlation between average daily step count and both ODI and SF-36 PCS scores. While this study included only a small sample of patients with relatively short-term follow-up, Scheer et al. reported that their findings may encourage future investigation of remote objective physical activity monitoring in the postoperative period.

\section{Discussion}

This systematic review of the literature highlights that while past TM utilization within spine surgery has been limited, there is a growing interest in this technology. Specifically, this systematic review identified 12 studies, including 4 RCTs, assessing the utility of TM compared to standard care for patients with spinal pathologies. Analysis of the included studies highlights that clinical applications of TM have predominately focused on enhancing perioperative communication between patients and their surgeons by taking advantage of modern mobile phone and online technologies. The use of TM for certain tasks such as postoperative surveys was preferred by patients for convenience, over in-person options.

\section{Current Use of TM in Spine Medicine}

The studies identified by this systematic review dealt with tools for patient communication and instructions. Whether surveying patient-reported functional status, improving preoperative compliance, or monitoring postoperative symptoms, these studies found that TM interventions were generally successful, and were met with high satisfaction from patients. The positive impact of TM in patient satisfaction is multifactorial but likely centers around increased convenience and enhanced communication. ${ }^{12,22}$ For example, the strongest predictors of patient 
TABLE 3. Results of the studies included in the systematic review

\begin{tabular}{|c|c|c|c|c|c|}
\hline $\begin{array}{l}\text { Authors } \\
\& \text { Year }\end{array}$ & $\begin{array}{l}\text { Sample } \\
\text { Size }\end{array}$ & Results & Conclusions & Limitations & $\begin{array}{l}\text { Quality } \\
\text { Rating }\end{array}$ \\
\hline $\begin{array}{l}\text { Bethune } \\
\text { et al., } \\
2018^{10}\end{array}$ & 38 & $\begin{array}{l}\text { Questionnaire scores significantly higher } \\
\text { in intervention group }\end{array}$ & $\begin{array}{l}\text { Use of e-book supplement } \\
\text { enhances knowledge retention in } \\
\text { pt standard consent process for } \\
\text { neurosurgical \& spinal } \\
\text { procedures }\end{array}$ & $\begin{array}{l}\text { Small sample size, lack of } \\
\text { comparison in baseline } \\
\text { characteristics such as education } \\
\text { level, bias toward intervention } \\
\text { group exposed to information twice } \\
\text { but control group only once }\end{array}$ & Fair \\
\hline $\begin{array}{l}\text { Bokshan } \\
\text { et al., } \\
2016^{11}\end{array}$ & 49 & $\begin{array}{l}\text { No significant difference btwn phone \& } \\
\text { in-person data for ODI \& SRS-22r; } \\
\text { intraclass correlation coefficient was } \\
0.91 \& 0.86 \text { for SRS-22r \& ODI }\end{array}$ & $\begin{array}{l}\text { Phone administration of SRS-22r } \\
\text { \& ODI for ASD pts was reliable } \\
\text { tool for disability assessment \& } \\
\text { preferred option for } 57 \% \text { of pts }\end{array}$ & $\begin{array}{l}\text { Sample size small, w/ variable } \\
\text { previous exposure to surveys }\end{array}$ & NA \\
\hline $\begin{array}{l}\text { Debono } \\
\text { et al., } \\
2016^{12}\end{array}$ & 60 & $\begin{array}{l}55 / 60 \text { pts use mobile app; during } 15 \\
\text { days postop, } 29 \text { alarms detected by } \\
\text { neurosurgical nurse; overall satisfaction } \\
\text { of mobile app } 3.5 / 4\end{array}$ & $\begin{array}{l}\text { Pts found mobile app useful, easy } \\
\text { to use; facilitated earlier return } \\
\text { discharge while minimizing need } \\
\text { for in-person visit }\end{array}$ & $\begin{array}{l}\text { No comparator, selective group of pts } \\
\text { w/ close follow-up w/ clinic nurses } \\
\text { may have biased pt experience } \\
\text { toward positive outcome }\end{array}$ & Fair \\
\hline $\begin{array}{l}\text { Dicianno } \\
\text { et al., } \\
2016^{17}\end{array}$ & 23 & $\begin{array}{l}\text { Pt physical independence, self-man- } \\
\text { agement skill, depression, \& HRQOL } \\
\text { did not differ btwn intervention group } \\
\text { \& control group. However, subset } \\
\text { of pts deemed high-volume user of } \\
\text { mobile app had higher scores for self- } \\
\text { management \& independent living. No } \\
\text { differences in medical events such as } \\
\text { emergency room visits or infection }\end{array}$ & $\begin{array}{l}\text { Use of mobile app for supporting } \\
\text { self-management in adults w/ } \\
\text { myelomeningocele is feasible. } \\
\text { However, positive impact of } \\
\text { mobile app only observed in } \\
\text { subset of high-volume users }\end{array}$ & $\begin{array}{l}\text { Small sample size w/ significant no. of } \\
\text { outcomes analyzed could increase } \\
\text { false-positive findings }\end{array}$ & Good \\
\hline $\begin{array}{l}\text { Felbaum } \\
\text { et al., } \\
2018^{13}\end{array}$ & 56 & $\begin{array}{l}\text { 54/56 pts downloaded \& used mobile app } \\
\text { to send postop wound images \& pain } \\
\text { scores; } 1 \text { postop complication (wound } \\
\text { infection) picked up via mobile app. No } \\
\text { op cancellations due to noncompliance } \\
\text { w/ preop instructions }\end{array}$ & $\begin{array}{l}\text { Mobile app for pre- \& postop } \\
\text { reminders \& follow-up is feasible } \\
\text { for spine procedures }\end{array}$ & $\begin{array}{l}\text { Small sample size, no comparison w/ } \\
\text { standard postop follow-up }\end{array}$ & Poor \\
\hline $\begin{array}{l}\text { Hou } \\
\text { et al., } \\
2019^{18}\end{array}$ & 168 & $\begin{array}{l}\text { Improvement of primary outcomes in } \\
\text { eHealth group superior to standard } \\
\text { care group at } 24 \text { mos postop }\end{array}$ & $\begin{array}{l}\text { Greater pain \& function improve- } \\
\text { ments in pts using mobile app } \\
\text { for postop rehab than pts not } \\
\text { participating in traditional rehab }\end{array}$ & $\begin{array}{l}\text { Specified rehab program not standard } \\
\text { care but rather informal instruction } \\
\text { on physical activity; control group's } \\
\text { physical activity not followed; } \\
\sim 30 \% \text { pt loss at 2-yr follow-up }\end{array}$ & Fair \\
\hline $\begin{array}{l}\text { Nitikman } \\
\text { et al., } \\
2017^{14}\end{array}$ & 96 & $\begin{array}{l}\text { No significant difference btwn groups } \\
\text { based on paper- vs internet-based } \\
\text { survey results; } 84 \% \text { of pts preferred } \\
\text { internet-based questionnaire }\end{array}$ & $\begin{array}{l}\text { SRS-30 \& PODCI survey collec- } \\
\text { tion via internet is reliable \& also } \\
\text { preferred method for adolescents } \\
\text { w/ idiopathic scoliosis }\end{array}$ & $\begin{array}{l}\sim 30 \% \text { pts did not complete follow-up } \\
\quad \text { survey }\end{array}$ & NA \\
\hline $\begin{array}{l}\text { Scheer } \\
\text { et al., } \\
2017^{21}\end{array}$ & 22 & $\begin{array}{l}\text { Average no. of daily steps correlated w/ } \\
\text { postop ODI \& SF-36 PCS scores at } 6 \\
\text { mos postop }\end{array}$ & $\begin{array}{l}\text { Postop physical activity level may } \\
\text { be associated w/ functional } \\
\text { outcomes; pilot study suggests } \\
\text { further investigation }\end{array}$ & $\begin{array}{l}\text { Small sample size, short-term follow- } \\
\text { up only, nonstandardized physical } \\
\text { activity regimen }\end{array}$ & \\
\hline $\begin{array}{l}\text { Stewart } \\
\text { et al., } \\
2019^{15}\end{array}$ & 174 & $\begin{array}{l}5.6 \% \text { cancellation rate in app nonusers vs } \\
\text { no case cancellations in app users }\end{array}$ & $\begin{array}{l}\text { Mobile app for preop instruc- } \\
\text { tions \& reminders increased pt } \\
\text { compliance \& decreased case } \\
\text { cancellation }\end{array}$ & $\begin{array}{l}\text { Small sample, nonrandomized group } \\
\text { assignment }\end{array}$ & Fair \\
\hline $\begin{array}{l}\text { Strøm } \\
\text { et al., } \\
2019^{16}\end{array}$ & 114 & $\begin{array}{l}\text { No significant change in HADS scores } \\
\text { in intervention or control group \& no } \\
\text { differences btwn groups; high rate of } \\
\text { compliance w/ online tool in interven- } \\
\text { tion group }\end{array}$ & $\begin{array}{l}\text { No significant impact on HADS } \\
\text { scores, but high compliance } \\
\text { indicates potential applicability in } \\
\text { this population }\end{array}$ & Small sample size & Fair \\
\hline
\end{tabular}


TABLE 3. Results of the studies included in the systematic review

\begin{tabular}{|c|c|c|c|c|c|}
\hline $\begin{array}{c}\text { Authors } \\
\& \text { Year }\end{array}$ & $\begin{array}{l}\text { Sample } \\
\text { Size }\end{array}$ & Results & Conclusions & Limitations & $\begin{array}{l}\text { Quality } \\
\text { Rating }\end{array}$ \\
\hline $\begin{array}{l}\text { Strøm } \\
\text { et al., } \\
2019^{20}\end{array}$ & 48 & $\begin{array}{l}\text { No sociodemographic factors associated } \\
\text { w/ support group use; women more } \\
\text { likely to be more active users; posts } \\
\text { w/in group categorized into } 7 \text { major } \\
\text { themes (e.g., pain management, rehab, } \\
\text { social experiences) }\end{array}$ & $\begin{array}{c}\text { No sociodemographic predictors of } \\
\text { support group use, difference in } \\
\text { degree of use in men vs women }\end{array}$ & $\begin{array}{l}\text { Small sample size, observational } \\
\text { design only, post hoc analysis of } \\
\text { RCT pt population }\end{array}$ & Fair \\
\hline $\begin{array}{l}\text { Thakar } \\
\text { et al., } \\
2018^{19}\end{array}$ & 1200 & $\begin{array}{l}\text { TM had overall higher utility ( } 89 \% \text { vs } 80 \% \text { ) } \\
\text { \& lower average absolute cost \& higher } \\
\text { cost effectiveness (ICER } 39,400 \text { Indian } \\
\text { rupees/unit effectiveness) }\end{array}$ & $\begin{array}{l}\text { Neurosurgical postop TM consulta- } \\
\text { tions are economically \& clinically } \\
\text { practical; TM is cost-effective } \\
\text { even w/ } 50 \% \text { unsuccessful } \\
\text { consultations }\end{array}$ & $\begin{array}{l}\text { No randomization (in-person groups } \\
\text { comprised pts who deferred TM } \\
\text { visit), disparate study group size } \\
\text { (in-person group only } 166 \text { pts), no. } \\
\text { of spine pts not reported, signifi- } \\
\text { cant mismatch in cranial/spine ratio } \\
\text { btwn groups (spine } 56 \% \text { of TM } \\
\text { group, } 90.9 \% \text { of in-person group) }\end{array}$ & NA \\
\hline
\end{tabular}

satisfaction after lumbar spine surgery were patients' perceptions of healthcare providers attempting to ease their pain, clarification on medication, and feeling that they are being heard. ${ }^{23}$ Debono et al. showed that a mobile app for postoperative follow-up can provide these requirements of positive patient experiences with prompt analgesia management, facilitating the transition to home, and preventing emergency room visits. ${ }^{12}$ By expanding the avenue for patient communication, TM may allow patients to feel heard without needing to visit the clinic, thus eliminating the hassles associated with in-person hospital visits, such as traffic, parking, and waiting rooms.

With an increasing emphasis on data-driven practices in spine surgery, the ability to obtain postoperative PROs (e.g., responses to ODI and SF-36 questionnaires) at clinically relevant time points without the need for patients to physically appear in the clinic may greatly improve overall data yields by making the process more convenient and mitigating the risk of losing data from missed appointments. ${ }^{11,14}$ Similarly, TM instruments for postoperative monitoring can automate the collection of postoperative pain scores and analgesic consumption-two common target variables in spine outcomes research. ${ }^{12,18}$ In the age of smartphones and fitness trackers, the potential of these tools to record kinematic data (in addition to heart rate and other vital signs already commonly captured by everyday devices) in real time throughout the day represents yet another potential expansion in real-time data collection through TM. ${ }^{21}$

While these improvements in data collection can be of immense academic benefit, the promise of TM may lie in enhancing the efficiency of outpatient clinical encounters and addressing barriers against access to care. With expected variance between regions and healthcare systems, TM visits are associated with overall cost reductions compared to traditional in-person office visits, which often require significant time and travel, office staff, and other resource investments. ${ }^{19}$ Furthermore, patients who may have relocated after surgery or who traveled for their sur- gery could remain in contact with their primary surgeon via TM rather than being forced to transfer their care as a function of geography rather than clinical need. Although conventional clinic visits will always be necessary (neurological examination, wound care, etc.), these virtual visits can significantly reduce the number of routine follow-up visits by shifting scheduled encounters to an in-person visit only when requested by the patients or deemed necessary from the TM visit.

\section{Future Direction of TM}

Perhaps the most salient question raised by this review is simply "where are the studies?" The paucity of research in this topic likely reflects the fact that, for various reasons, TM has not been widely adopted in spine surgery. These include the inherent limitations of TM visits for physical examination, previous restrictions on billing, the frequent need for technical troubleshooting, and a prior lack of an impetus to change. Studies in other fields, such as physiatry and neurology, may provide solutions to address limitations of $\mathrm{TM}$, such as the limited ability to perform physical examination. ${ }^{24-26}$ For example, studies have shown the feasibility and accuracy of performing a Unified Parkinson's Disease Rating Scale motor assessment using TM in patients with Parkinson's disease. ${ }^{26-28}$ Other potential solutions utilize sensors in smartphones to measure certain components of patient assessments, such as range of motion, tremor, hand dexterity, and gait. . $2,29,30^{2}$ However, at this time, the advantages of TM in neurosurgery are most noticeable for postoperative follow-up, where patient-physician rapport is established, baseline neurological function is documented, and subtle neurological findings are not as essential in establishing management plans. For new surgical consultations, TM may be utilized as an adjunct to in-person visits for triaging of referral and follow-up discussions regarding treatment plans. During the COVID-19 period, TM in our institution was often used to triage urgent cases with progressive neurological deficits, along with ensuring continuing postop- 
erative follow-up. Because the use of TM is becoming part of daily practice, this systematic review highlights several considerations for future applications and investigations of TM in spine surgery.

All of the studies of TM interventions identified in our review describe experimentation performed in a controlled, planned fashion. Surgeons and clinics had advanced notice of the new practices. Patients could be selected for TM and-importantly-could opt out if desired. In short, these studies describe TM adoption by choice, whereas our community is currently facing TM implementation by necessity. This novel practice environment may, therefore, impact patient satisfaction and participation, thereby affecting outcomes. Studies examining the user experiences of various stakeholders, including healthcare providers and patients, during the COVID-19 period may provide greater insight into the strengths and limitations of current TM options.

Future studies focusing on economic evaluations of TM are also important in validating the utility of this technology. While Thakar et al. did demonstrate a significant cost-saving benefit with TM visits, ${ }^{19}$ their study was performed outside of North America. Due to the variability in healthcare systems around the world, such as single versus multipayer systems and for-profit versus nonprofit institutions, the generalizability of the findings of Thakar et al. is limited when considering the United States healthcare system. However, the high savings margin they report ( $>50 \%$ cost reduction in the TM group) supports the importance of examining the cost-effectiveness of TM. As more corporate TM services become available, incorporating the cost of infrastructure and software into the economic analysis will be essential. Research collaboration with these companies may provide a wealth of data that can further enhance the utilization and quality of TM for patients. In addition, establishing evidence of the cost-effectiveness of TM will aid in addressing administrative and legal concerns, such as insurance coverage and liability. These investigations will be particularly useful as hospitals begin to evaluate the economic impact of COVID-19 and potential means to overcome their financial burdens.

While previous studies frequently comment on patient satisfaction, assessing physician satisfaction with TM will be critical in considering the long-term use of TM in spine surgery. Surgeons may be concerned about the potential loss of patient rapport afforded by an in-person clinic visit, which enables a deeper understanding of patients' perceptions of their diseases and realistic expectations for surgery. This is particularly important because treatment decisions for patients with spine pathology are nuanced and individualized-often based on subtle clinical findings and discussions with the patients.

\section{Study Limitations}

As noted in this systematic review, the availability of studies on TM in spine surgery is limited, and therefore only descriptive analysis was performed. Furthermore, due to the heterogeneity of the included studies, a quantitative pooled analysis or other method of meta-analysis was not possible in the present review. Due to the native language of the authors, this search was restricted to English- language publications, which may result in missed data from foreign-language publications.

\section{Conclusions}

This systematic review of the literature identified 12 studies exploring the application of TM in spine surgery. In general, TM interventions were used to facilitate communication between patients and surgeons and were successful in their early implementation. The current rise in TM adoption due to the COVID-19 crisis presents an opportunity to further develop and validate this technology. In turn, the data generated in the coming months will inform future applications of TM in spine surgery.

\section{Appendix}

\section{Summary of Systematic Literature Search Terms \\ Theme: Telemedicine}

mHealth, eHealth, telecommunication, telemedicine, telehealth, virtual health, virtual medicine, remote consultation, videoconferencing, mobile, internet, SMS [short message service], text, message, messaging, smartphone

\section{Theme: Spine Surgery}

spine surgery, spinal surgery, spinal procedure, spine, lumbar, cervical spine [used in lieu of "cervical" alone to avoid gynecologic literature], thoracic, sacral

\section{Acknowledgments}

We would like to acknowledge the Rush University Department of Neurological Science Research Team for their help in this study.

\section{References}

1. de la Torre-Díez I, López-Coronado M, Vaca C, et al. Costutility and cost-effectiveness studies of telemedicine, electronic, and mobile health systems in the literature: a systematic review. Telemed J E Health. 2015;21(2):81-85.

2. Kruse CS, Krowski N, Rodriguez B, et al. Telehealth and patient satisfaction: a systematic review and narrative analysis. BMJ Open. 2017;7(8):e016242.

3. Dorsey ER, Topol EJ. State of telehealth. $N$ Engl J Med. 2016;375(2):154-161.

4. Greven ACM, Rich CW, Malcolm JG, et al. Letter: Neurosurgical management of spinal pathology via telemedicine during the COVID-19 pandemic: early experience and unique challenges. Neurosurgery. 87(2):E192-E196.

5. LoPresti MA, McDeavitt JT, Wade K, et al. Letter: Telemedicine in neurosurgery-a timely review. Neurosurgery. 2020; 87(2):E208-E210.

6. Liberati A, Altman DG, Tetzlaff J, et al. The PRISMA statement for reporting systematic reviews and meta-analyses of studies that evaluate health care interventions: explanation and elaboration. PLoS Med. 2009;6(7):e1000100.

7. Niknamian S. Systematic review on tele-wound-care in spinal cord injury (SCI) patients and the impact of telemedicine in decreasing the cost. Phys Med Rehabil Res. 2019;4: 10.15761/PMRR.1000199.

8. NIH National Heart Lung and Blood Institute. Study quality assessment tools. Accessed July 27, 2020. https://www.nhlbi. nih.gov/health-topics/study-quality-assessment-tools

9. Moher D, Liberati A, Tetzlaff J, Altman DG. Preferred reporting items for systematic reviews and meta-analyses: the PRISMA statement. PLoS Med. 2009;6(7):e1000097. 
10. Bethune A, Davila-Foyo M, Valli M, da Costa L. e-Consent: approaching surgical consent with mobile technology. Can J Surg. 2018;61(5):339-344.

11. Bokshan SL, Godzik J, Dalton J, et al. Reliability of the revised Scoliosis Research Society-22 and Oswestry Disability Index (ODI) questionnaires in adult spinal deformity when administered by telephone. Spine J. 2016;16(9):1042-1046.

12. Debono B, Bousquet P, Sabatier P, et al. Postoperative monitoring with a mobile application after ambulatory lumbar discectomy: an effective tool for spine surgeons. Eur Spine J. 2016;25(11):3536-3542.

13. Felbaum DR, Stewart JJ, Anaizi AN, et al. Implementation and evaluation of a smartphone application for the perioperative care of neurosurgery patients at an academic medical center: implications for patient satisfaction, surgery cancelations, and readmissions. Oper Neurosurg (Hagerstown). 2018;14(3):303-311.

14. Nitikman M, Mulpuri K, Reilly CW. Internet-administered health-related quality of life questionnaires compared with pen and paper in an adolescent scoliosis population: a randomized crossover study. J Pediatr Orthop. 2017;37(2):e75e79.

15. Stewart JJ, Fayed I, Henault S, et al. Use of a smartphone application for spine surgery improves patient adherence with preoperative instructions and decreases last-minute surgery cancellations. Cureus. 2019;11(3):e4192.

16. Strøm J, Nielsen CV, Jørgensen LB, et al. A web-based platform to accommodate symptoms of anxiety and depression by featuring social interaction and animated information in patients undergoing lumbar spine fusion: a randomized clinical trial. Spine J. 2019;19(5):827-839.

17. Dicianno BE, Fairman AD, McCue M, et al. Feasibility of using mobile health to promote self-management in spina bifida. Am J Phys Med Rehabil. 2016;95(6):425-437.

18. Hou J, Yang R, Yang Y, et al. The effectiveness and safety of utilizing mobile phone-based programs for rehabilitation after lumbar spinal surgery: multicenter, prospective randomized controlled trial. JMIR Mhealth Uhealth. 2019;7(2): e10201.

19. Thakar S, Rajagopal N, Mani S, et al. Comparison of telemedicine with in-person care for follow-up after elective neurosurgery: results of a cost-effectiveness analysis of 1200 patients using patient-perceived utility scores. Neurosurg Focus. 2018;44(5):E17.

20. Strøm J, Høybye MT, Laursen M, et al. Lumbar spine fusion patients' use of an internet support group: mixed methods study. J Med Internet Res. 2019;21(7):e9805.

21. Scheer JK, Bakhsheshian J, Keefe MK, et al. Initial experience with real-time continuous physical activity monitoring in patients undergoing spine surgery. Clin Spine Surg. 2017; 30(10):E1434-E1443.

22. Wilkinson JR, Spindler M, Wood SM, et al. High patient satisfaction with telehealth in Parkinson disease: a randomized controlled study. Neurol Clin Pract. 2016;6(3):241-251.

23. Levin JM, Winkelman RD, Tanenbaum JE, et al. Key drivers of patient satisfaction in lumbar spine surgery. $J$ Neurosurg Spine. 2018;28(6):586-592.
24. Cottrell MA, O'Leary SP, Swete-Kelly P, et al. Agreement between telehealth and in-person assessment of patients with chronic musculoskeletal conditions presenting to an advanced-practice physiotherapy screening clinic. Musculoskelet Sci Pract. 2018;38:99-105.

25. Peterson S, Kuntz C, Roush J. Use of a modified treatmentbased classification system for subgrouping patients with low back pain: Agreement between telerehabilitation and faceto-face assessments. Physiother Theory Pract. 2019;35(11): 1078-1086.

26. Schneider RB, Biglan KM. The promise of telemedicine for chronic neurological disorders: the example of Parkinson's disease. Lancet Neurol. 2017;16(7):541-551.

27. Dorsey ER, Venkataraman V, Grana MJ, et al. Randomized controlled clinical trial of "virtual house calls" for Parkinson disease. JAMA Neurol. 2013;70(5):565-570.

28. Abdolahi A, Scoglio N, Killoran A, et al. Potential reliability and validity of a modified version of the Unified Parkinson's Disease Rating Scale that could be administered remotely. Parkinsonism Relat Disord. 2013;19(2):218-221.

29. Pourahmadi MR, Bagheri R, Taghipour M, et al. A new iPhone application for measuring active craniocervical range of motion in patients with non-specific neck pain: a reliability and validity study. Spine J. 2018;18(3):447-457.

30. Rye Hanton C, Kwon YJ, Aung T, et al. Mobile phone-based measures of activity, step count, and gait speed: results from a study of older ambulatory adults in a naturalistic setting. JMIR Mhealth Uhealth. 2017;5(10):e104.

\section{Disclosures}

The authors report no conflict of interest concerning the materials or methods used in this study or the findings specified in this paper.

\section{Author Contributions}

Conception and design: Ryu, Traynelis. Acquisition of data: Kolcun, Ryu. Analysis and interpretation of data: Kolcun, Ryu, Traynelis. Drafting the article: Kolcun, Ryu. Critically revising the article: Kolcun, Ryu, Traynelis. Reviewed submitted version of the manuscript: Kolcun, Ryu, Traynelis. Approved final version of the manuscript on behalf of all authors: Traynelis. Administrative/ technical/material support: Traynelis. Study supervision: Traynelis.

\section{Correspondence}

Vincent C. Traynelis: Rush University Medical Center, Chicago, IL.vincent_traynelis@rush.edu. 